\title{
Welfare Deprivation of Women in Rural North-West, Nigeria
}

\author{
Oladokun Yetunde Olasimbo Mary ${ }^{1}$, Kemisola Omorinre Adenegan ${ }^{1}$, Isaac Busayo Oluwatayo ${ }^{2}$ \\ ${ }^{1}$ Department of Agricultural Economics, Faculty of Agriculture, University of Ibadan, Ibadan, Nigeria \\ ${ }^{2}$ Department of Agricultural Economics and Animal Production, Faculty of Agriculture, University of Limpopo, Limpopo, South Africa
}

Email address:

yetunde.oladokun@gmail.com (O.Y. O. Mary)

To cite this article:

Oladokun Yetunde Olasimbo Mary, Kemisola Omorinre Adenegan, Isaac Busayo Oluwatayo. Welfare Deprivation of Women in Rural NorthWest, Nigeria. European Business \& Management. Vol. 4, No. 1, 2018, pp. 9-17. doi: 10.11648/j.ebm.20180401.12

Received: October 1, 2017; Accepted: November 11, 2017; Published: December 14, 2017

\begin{abstract}
Women are important segment of the human population and appropriate investment in their welfare would be of great benefit at individual, household, and community levels. There has been an increasing incidence of welfare deprivation among women in rural Nigeria. Thus this study examined the welfare deprivation of women in rural North-West (NW) Nigeria. Secondary data from Nigeria Demographic Health Survey (NDHS, 2013) was used for this study and 6798 rural women were sampled in the survey. Data analysis was done using descriptive statistics, fuzzy analysis and logit regression. The mean age of women in NW is 30 years and a standard deviation of 9. Majority of the women in NW had no formal education (85.80\%) and this is a major contributor to women's welfare. Women in this region have a mean household size of 7 and this had an effect on their deprivation status. The Deprivation Index for rural women ranges from 0.01 to 0.82 with a mean value of 0.30 . Educational attainment, employment type, household size, marital status, age of the woman, state of residence determines a woman's deprivation status in rural NW. The study concluded that women are deprived in rural NW. Government and nonGovernmental Organisations should put in place measures to augment human capacity development of the women in NW through increased school funding in order to overcome the financial constraints of parents involvement in educating up-coming generations.
\end{abstract}

Keywords: Asset, Deprivation, North-West Nigeria, Welfare, Women

\section{Introduction}

Deprivation is a state of observable and demonstrable disadvantage relative to the local community or the wider society or nation to which the individual, family or group belongs [20] [18]. This disadvantage may be with regard to food, clothing, housing condition, or lack of education and exclusion from the decision- making class among others. A person is therefore considered deprived to the extent that he falls short of the level attained by his contemporaries within the same society, or below generally acceptable social status $[19 ; 16]$. Women play a very vital role in the development of communities and nations. Development is incomplete if it fails to comprehend the contributions of women [6]. Women in the North-West are particularly deprived compared to their counterpart in the south as a result of socio cultural norms and beliefs in their area where women are seen and treated as second class citizens. Women are married off early forfeiting their education and do not acquire the required skill to help them in life. They are particularly dependent on their husbands who has to take care of three or four of such women and their children. Thus women are limited and they continually remain in poor. Welfare among women is determined not only by their health status, but from other dimensions like education, food and nutrition, housing and sanitation characteristics, asset ownership, autonomy, type of employment, information access, and others.

Several studies had considered the issue of welfare deprivation at individual and household level. [13] examined the level of welfare deprivation among riverine households in Southwestern Nigeria using five dimensions consisting of sixteen welfare indicators. Using the principal component analysis, the deprivation index of the households ware aggregated into a three-component deprivation structure, namely: housing condition deprivation; health and nutrition deprivation; and social network deprivation. Considering the deprivation characteristics of the riverine households, access to public basic educational facilities as well as other stable 
means of income than farming, fishing and natural resource collection activities- should be put in place to reduce their deprivation level in many of the indicated welfare dimensions.

Also, in the study on Gender Inequality in Multidimensional Welfare Deprivation in West Africa by [3]. The study made use of a counting approach to examine gender issues in Burkina Faso and Togo using household surveys, namely Enquête Intégrale surles Conditions de Vie des Ménages (2009/2010) and Core Welfare Indicators Questionnaire (2011), respectively. It focused on six dimensions (housing, basic utilities, assets, education, employment and access to credit) largely recognized as MDG targets. Their findings indicated that overall individuals are the most deprived in education in Burkina Faso, while the reverse situation is true in Togo. Gender inequality was observed in all dimensions since women always seem to be more deprived than men. The situation was also marked by regional disparities. Moreover, the assessment of dimensional contributions showed different patterns for each country. While employment proves to be the main contributor of gender inequality in Burkina Faso, three dimensions (assets, access to credit and employment) accounted for most of the total contribution to gender inequality in Togo.

Poverty and deprivation is one of the fundamental challenges of our contemporary world [17]. [5] revealed that more than a billion people live below US\$1.25 per day globally while about 1.75 billion persons suffer from multidimensional poverty, with deprivations in heath, economic opportunities, education, and living standard. Greater proportion of poor people is found in developing countries, with Africa accounting for the largest. Several countries across the globe are narrowing the rich-poor gap; however, the gap appears to be widening in Africa. [7] noted that "poverty, slow economic growth, and unequal income and wealth distribution are endemic in African countries". Nigeria, the largest Black country in the world, is characterized by high rate of poverty despite her economic fortune [17]. According to [11], the incidence of poverty in Nigeria increased from about $27.2 \%$ in 1980 to $46.3 \%$ in 1985 . It decreased marginally to $42.7 \%$ in 1992 and rose to $65.6 \%$ in 1996 . Conversely, the rate declined to $54.4 \%$ in 2004 and increased again to $69.0 \%$ in 2010 . The proportion of the Nigerian population living in absolute poverty rose from $54.7 \%$ in 2004 to $60.9 \%$ in 2010 . It is in view of this that this study proffered answers to these research question

1. What is the welfare deprivation status of women in North West?

2. What are the factors influencing their deprivation status?

Objectives

The main objective of this study is to estimate the welfare deprivation of women in rural North-West Nigeria. It was achieved through the following research question

1. To analyse the welfare deprivation status of women in North West.

2. To determine the factors influencing their deprivation status

\section{Method}

\subsection{Study Area}

The area of study for this research is rural North-West Nigeria. North- West geopolitical zone is one of the six geopolitical zones in Nigeria. Secondary data from Nigeria Demographic Health Survey [12] was used for this study. The sample for the 2013 NDHS was a stratified sample, selected independently in three stages from the sampling frame. Stratification was achieved by separating each state into urban and rural areas. Information from 6798 women were used for this study.

\subsection{Analytical Procedure}

Descriptive statistics fuzzy set theory and logit regression were used in this study. The descriptive statistics used include percentages, frequency distribution tables, and the mean.

\subsubsection{Fuzzy Set Analysis}

This was used to estimate the deprivation status of women. The fuzzy set substitutes the characteristic function of a crisp set that assigns a value of 1 or 0 . Large values denote high degree of membership. $[9 ; 8]$. The degree of deprivation is shown by the placement of the individual on the 0 or 1 value or other values in-between. The model is considered as follows:

Assume a population $\mathrm{A}$ of $\mathrm{n}$ individuals, $\mathrm{A}=\left(\mathrm{a}_{1}, \mathrm{a}_{2}\right.$, $\left.a_{3} \ldots a_{n}\right)$. A fuzzy subset $B$ includes all individuals with aic $B$. The degree of deprivation of the ith individual $(i=1, \ldots, n)$ with respect to a particular attribute $j$ given that $(j=1, \ldots \ldots$, $\mathrm{m})$ is defined as:

$$
\mu_{\beta \mid} x j(a i) \mid=x i j, 0 \leq x i j \leq 1 \text { where: }
$$

$x_{i j}=1$; condition of total lack of welfare attribute (state of deprivation)

$x_{i j}=0$; condition of full possession of welfare attribute

$0 \leq \mathrm{x}_{i j} \leq 1$; conditions within the range of lack and full possession

The variables that define indicators of welfare are either dichotomous or categorical in nature.

Dichotomous Variables

These are answered by either Yes or No; with the yes being a state of wellbeing and the No, a state of deprivation. Following [14], from a universal set of A individuals, we define the membership function of fuzzy subset of B for the ith individual $(\mathrm{i}=1 \ldots \mathrm{n})$ that possesses the $\mathrm{jth}$ welfare attribute $(\mathrm{j}=1 \ldots \ldots \mathrm{m})$ as:

$$
\mu_{\beta \mid} x j(a i) \mid=\mathrm{xij},
$$

$\mathrm{X} \mathrm{j}$ (ai) is the $\mathrm{m}$ order of welfare attributes that will result in a state of wellbeing if totally or partially owned by the ith woman.

$x i j=1$, if the ith individual possesses the $j$ th attribute

$x i j=0$, if the ith individual does not possess the jth wellbeing attribute.

Categorical Variables

Categorical variables present themselves in a range of 
values, rather than just two values. Expressing the membership function for these variables take the form:

$$
\mu_{\beta \mid} x j(a i) \mid=\mathrm{xij}
$$

Where $C_{\max }$ is the value that depicts high level of deprivation in the $j^{\text {th }}$ attributes, which translates to lowest level of welfare; while $C \mathrm{~min}$ is the lowest level of deprivation in the $j^{\text {th }}$ attribute which indicates highest level of welfare in the $a i^{\text {th }}$ woman. Thus, the modalities are arranged in decreasing order of welfare attainment $C_{i j}$ values are the intermediate values within the two thresholds, which depicts the position of the $a i^{\text {th }}$ woman within the modalities set forth.

$$
x i j=\mathrm{C} \text { min- } \mathrm{Ci} / \mathrm{C} \min -\mathrm{Cmax}, \text { if } \mathrm{Cmax} \leq \mathrm{Cij} \leq \mathrm{Cmin}
$$

so that $0 \leq x i j \leq 1$

In specifying the well-being index for the population of women, following $[15,14,2]$ it is expressed as:

$$
\mu \beta\left(a_{i}\right)=\sum_{j=1}^{n} x_{i j} w_{j} \mid \sum_{j=1}^{m} w_{j}
$$

Where wj is the weight given to the jth attribute. $\mu \beta$ (ai) measures the degree of well-being of the ith individual as a weighting function of $\mathrm{m}$ attributes/ indicators.

Selected Dimensions and Methods of Evaluation are

\begin{tabular}{|c|c|c|}
\hline Indicator & Selected criteria & Deprivation \\
\hline \multicolumn{3}{|l|}{ Housing and Sanitation } \\
\hline Source of drinking water & Pipe borne water and treated $1=$ improved, $0=$ otherwise & $0=$ non deprived, $1=$ deprived \\
\hline Toilet facility & $1=$ improved, $0=$ otherwise & $0=$ non deprived, $1=$ deprived \\
\hline Main floor material & $1=$ improved, $0=$ otherwise & $0=$ non deprived, $1=$ deprived \\
\hline Main wall material & $1=$ use of finished material, $0=$ otherwise & $0=$ non deprived, $1=$ deprived \\
\hline Main roof material & $1=$ use of finished product, $0=$ otherwise & $0=$ non deprived, $1=$ deprived \\
\hline \multicolumn{3}{|l|}{ Autonomy } \\
\hline \multirow{2}{*}{$\begin{array}{l}\text { Final say on travel to market and outside } \\
\text { village/community }\end{array}$} & Women and husband take decision $=3$ & \multirow{2}{*}{$0=$ non deprived, $1=$ deprived } \\
\hline & $\begin{array}{l}\text { Women take decisions with another person }=2 \\
\text { Women take decisions alone }=1\end{array}$ & \\
\hline Final say on own health & Same as above & $0=$ non deprived, $1=$ deprived \\
\hline Final say on visit to friends and relatives & Same as above & $0=$ non deprived, $1=$ deprived \\
\hline Final say on making large household purchases & Same as above & $0=$ non deprived, $1=$ deprived \\
\hline Final say on money spending. & Same as above & $0=$ non deprived, $1=$ deprived \\
\hline Final say on husband's earnings & Same as above & $0=$ non deprived, $1=$ deprived \\
\hline \multicolumn{3}{|l|}{ Health and Nutrition } \\
\hline Place of delivery & Deliver in health facility $=1.0=$ otherwise & $0=$ non deprived, $1=$ deprived \\
\hline Antenatal care & Receive ante natal care from skilled attendant $=1,0=$ otherwise & $0=$ non deprived, $1=$ deprived \\
\hline Skilled attendant during delivery & $\begin{array}{l}\text { Attended to by skilled attendant during delivery }=1 \text {, } \\
0=\text { otherwise }\end{array}$ & $0=$ non deprived, $1=$ deprived \\
\hline & $18.5 \mathrm{~kg} / \mathrm{m}^{2}$ to $25.0 \mathrm{~kg} / \mathrm{m}^{2}=1$ & $0=$ non deprived, $1=$ deprived \\
\hline Body Mass Index (BMI) & $<18.5 \mathrm{~kg} / \mathrm{m}^{2}$ and $>25.0 \mathrm{~kg} / \mathrm{m}^{2}=0$ & \\
\hline \multicolumn{3}{|l|}{ Education } \\
\hline & woman with no formal education $=4$ & \\
\hline level of educational attainment & $\begin{array}{l}\text { woman with primary education }=3 \\
\text { woman with secondary education }=2\end{array}$ & $0=$ non deprived, $1=$ deprived \\
\hline & woman with tertiary education $=1$ & \\
\hline Literacy & $\begin{array}{l}\text { Women who can read part of a sentence or a whole sentence will be } \\
\text { regarded as literate. A value of } 1 \text { will be assigned, } 0=\text { otherwise }\end{array}$ & $0=$ non deprived, $1=$ deprived \\
\hline \multicolumn{3}{|l|}{ Employment } \\
\hline \multirow{5}{*}{$\begin{array}{l}\text { Employment status } \\
\text { Employment type }\end{array}$} & Currently employed $=1,0=$ otherwise & \multirow{5}{*}{$0=$ non deprived, $1=$ deprived } \\
\hline & Unemployed $=4$ & \\
\hline & Skilled and Unskilled manual employment $=3$ & \\
\hline & Agricultural and allied sector $=2$ & \\
\hline & Non Agriculture $=1$ & \\
\hline
\end{tabular}
presented in table 1 were used for this study.

Table 1. Selected Dimensions and Method of Evaluation.

Source: Authors' computations from the 2013 DHS data.

\subsubsection{Logit Regression}

Logit regression model was used to achieve objective two which is to determine the factors influencing the deprivation status of women. This identified the variables that significantly influence asset ownership by women. Logit regression analysis extends the techniques of multiple regression analysis to research situations in which the outcome variable is categorical. The model for logistic regression analysis assumes that the outcome variable $\mathrm{Z}$ is categorical (e. g. dichotomous) and models the probabilities associated with the value of $\mathrm{Z}$. The dependent Variable (Y) is dichotomous and takes the value 1 for the deprived individual and 0 for the non-deprived individual.

The logit model postulates the probability $\left(\mathrm{P}_{\mathrm{i}}\right)$ that a woman's deprivation status is a function of an index $\left(\mathrm{Z}_{\mathrm{i}}\right)$

Where:

$\left(Z_{i}\right)$ is an inverse of the standard logistic cumulative function of $\mathrm{P}_{\mathrm{i}}$ i. e. $P_{i}(y)=f\left(Z_{i}\right)$ 
$\left(Z_{i}\right)$ is also an inverse of the standard logistic cumulative function of $\mathrm{P}_{\mathrm{i}}$

i. e.

$$
P_{i}(y=1)=f\left(Z_{i}\right)
$$

The probability of a woman deprivation status is given by

$$
P_{i}(y=1)=\left(\frac{1}{1+e}\right)^{-Z_{i}}
$$

$e$ represents the base of natural logarithms (2.718)

The probability that a woman owns asset is calculated from $Z_{\mathrm{i}}$ value

$$
\mathrm{Z}_{\mathrm{i}}=\mathrm{b}_{0}+\mathrm{b}_{1} \mathrm{x}_{1}+\mathrm{b}_{2} \mathrm{x}_{2}+\mathrm{b}_{3} \mathrm{x}_{3}+\ldots \ldots \ldots+\mathrm{b}_{\mathrm{n}} \mathrm{x}_{\mathrm{n}}+\mu_{\mathrm{i}}
$$

$\mathrm{x}_{1}-\mathrm{X}_{\mathrm{n}}$ are the independent variables

$\mathrm{Z}_{\mathrm{i}}=$ asset ownership of rural woman $(1=$ asset index is greater than mean index, 0 otherwise)

$\mathrm{b}_{0}=$ constant

$\mathrm{b}_{1}=$ is the coefficient of the $\mathrm{X}$ 's variables.

$\mathrm{Z}_{\mathrm{i}}=$ probability of owning asset

$\mathrm{x}_{\mathrm{i}}$ represent vector of the explanatory variables for $i=$ $1 \ldots \ldots . n$ where $\mathrm{n}=1 \ldots \ldots . .11$

$\mu_{\mathrm{i}}$ represents the independent distributed error term

$\mathrm{x}_{1}=$ age $(1=15-24,2=25-34,3=35-49)$

level of educational attainment $(1=$ no education, $2=$ incomplete primary, $3=$ complete primary, $4=$ incomplete secondary, $5=$ complete secondary, $6=$ higher)

$\mathrm{x}_{3}=$ household size $(1=1-5,2=6-10,3=>10)$

$\mathrm{x}_{4}=$ marital status ( 1 if married 0 otherwise)

$\mathrm{x}_{5}=$ employment status (employed $=1$, unemployed $=0$ )

$\mathrm{x}_{7}=$ gender of household head (Male $=1$, female $=0$ )

$\mathrm{x}_{9}=$ Employment type of women (1=unemployed, $2=$ skilled and unskilled, $3=$ agriculture sector, $4=$ non agric. sector)

\section{Results}

\begin{tabular}{|c|c|c|}
\hline Variable & Frequency & Percentage \\
\hline \multicolumn{3}{|l|}{ Age } \\
\hline $15-24$ & 2169 & 31.91 \\
\hline $25-34$ & 2343 & 34.47 \\
\hline $35-49$ & 2286 & 33.63 \\
\hline Mean & 30 & \\
\hline Standard deviation & 9 & \\
\hline \multicolumn{3}{|l|}{ Educational attainment } \\
\hline No education & 5833 & 85.80 \\
\hline Incomplete primary & 203 & 2.99 \\
\hline Complete primary & 434 & 6.38 \\
\hline Incomplete secondary & 163 & 2.40 \\
\hline Complete secondary & 140 & 2.06 \\
\hline Higher & 25 & 0.37 \\
\hline \multicolumn{3}{|l|}{ Household size } \\
\hline $1-5$ & 2537 & 37.32 \\
\hline $6-10$ & 3082 & 45.34 \\
\hline$>10 f$ & 1179 & 17.34 \\
\hline Mean & 7 & \\
\hline Standard Deviation & 4 & \\
\hline \multicolumn{3}{|l|}{ Marital status } \\
\hline Single & 161 & 2.37 \\
\hline Married & 6637 & 97.63 \\
\hline \multicolumn{3}{|c|}{ Relationship to household head } \\
\hline Head & 195 & 2.87 \\
\hline Wife & 6233 & 91.69 \\
\hline Daughter and others & 370 & 5.44 \\
\hline \multicolumn{3}{|l|}{ Employment } \\
\hline Unemployed & 2591 & 38.11 \\
\hline Skilled and Unskilled & 960 & 14.12 \\
\hline Agriculture and allied & 150 & 2.21 \\
\hline Services & 3097 & 45.56 \\
\hline
\end{tabular}

Table 2. Distribution of women according to their Socio economic characteristics in rural North West Nigeria.

\begin{tabular}{|c|c|c|c|c|c|c|c|c|c|c|c|c|c|c|}
\hline \multirow{2}{*}{ Variable } & \multicolumn{2}{|c|}{ Jigawa } & \multicolumn{2}{|c|}{ Kaduna } & \multicolumn{2}{|l|}{ Kano } & \multicolumn{2}{|c|}{ Kastina } & \multicolumn{2}{|l|}{ Kebbi } & \multicolumn{2}{|c|}{ Sokoto } & \multicolumn{2}{|c|}{ Zamfara } \\
\hline & Freq & $\%$ & Freq & $\%$ & Freq & $\%$ & Freq & $\%$ & Freq & $\%$ & Freq & $\%$ & Freq & $\%$ \\
\hline \multicolumn{15}{|l|}{ Age } \\
\hline $15-24$ & 354 & 32.90 & 204 & 34.23 & 387 & 30.07 & 318 & 31.93 & 292 & 30.64 & 299 & 31.64 & 315 & 33.33 \\
\hline $25-34$ & 380 & 35.32 & 190 & 31.88 & 415 & 32.25 & 354 & 35.54 & 337 & 35.36 & 313 & 33.12 & 354 & 37.46 \\
\hline $35-49$ & 342 & 31.78 & 202 & 33.89 & 485 & 37.68 & 324 & 3.53 & 324 & 34.00 & 333 & 35.24 & 276 & 29.21 \\
\hline Mean & 29 & & 29 & & 30 & & 30 & & 30 & & 30 & & 29 & \\
\hline Standard deviation & 9 & & 9 & & 10 & & 9 & & 9 & & 9 & & 9 & \\
\hline \multicolumn{15}{|l|}{ Educational attainment } \\
\hline No education & 958 & 89.03 & 386 & 64.77 & 1028 & 79.88 & 821 & 82.43 & 863 & 90.56 & 893 & 94.50 & 884 & 93.54 \\
\hline Incomplete primary & 44 & 4.09 & 27 & 4.53 & 60 & 4.66 & 14 & 1.41 & 24 & 2.52 & 14 & 1.48 & 20 & 2.12 \\
\hline Complete primary & 46 & 4.28 & 67 & 11.24 & 119 & 9.25 & 115 & 11.55 & 44 & 4.62 & 15 & 1.59 & 28 & 2.96 \\
\hline Incomplete secondary & 22 & 2.04 & 57 & 9.56 & 41 & 3.19 & 17 & 1.71 & 12 & 1.26 & 8 & 0.85 & 6 & 0.63 \\
\hline Complete secondary & 6 & 0.56 & 44 & 7.38 & 35 & 2.72 & 26 & 2.61 & 10 & 1.05 & 14 & 1.48 & 5 & 0.53 \\
\hline Higher & 0 & 0.00 & 15 & 2.52 & 4 & 0.31 & 3 & 0.30 & 0 & 0.00 & 1 & 0.11 & 2 & 0.21 \\
\hline \multicolumn{15}{|l|}{ Household size } \\
\hline $1-5$ & 411 & 38.20 & 230 & 38.59 & 432 & 33.57 & 12 & 1.20 & 352 & 36.94 & 399 & 42.22 & 374 & 39.58 \\
\hline $6-10$ & 478 & 44.42 & 263 & 44.13 & 597 & 46.39 & 954 & 95.78 & 444 & 46.59 & 417 & 44.13 & 418 & 44.23 \\
\hline$>10$ & 187 & 17.38 & 103 & 17.28 & 258 & 20.05 & 30 & 3.01 & 157 & 16.47 & 129 & 13.65 & 153 & 16.19 \\
\hline Mean & 7 & & 7 & & 8 & & 7 & & 7 & & 7 & & 7 & \\
\hline Standard deviation & 4 & & 4 & & 4 & & 4 & & 4 & & 4 & & 4 & \\
\hline \multicolumn{15}{|l|}{ Marital status } \\
\hline Single & 32 & 11.02 & 23 & 3.86 & 29 & 2.25 & 27 & 2.71 & 23 & 2.41 & 16 & 1.69 & 11 & 1.16 \\
\hline Married & 1044 & 88.98 & 573 & 96.14 & 1258 & 97.75 & 969 & 97.29 & 930 & 97.59 & 929 & 98.31 & 934 & 98.84 \\
\hline $\begin{array}{l}\text { Relationship to } \\
\text { household head }\end{array}$ & & & & & & & & & & & & & & \\
\hline
\end{tabular}

Source: Authors' computations from the 2013 DHS data.

Table 3. Distribution of women by their socio economic characteristics across states in rural North West. 


\begin{tabular}{|c|c|c|c|c|c|c|c|c|c|c|c|c|c|c|}
\hline \multirow{2}{*}{ Variable } & \multicolumn{2}{|c|}{ Jigawa } & \multicolumn{2}{|c|}{ Kaduna } & \multicolumn{2}{|l|}{ Kano } & \multicolumn{2}{|c|}{ Kastina } & \multicolumn{2}{|c|}{ Kebbi } & \multicolumn{2}{|l|}{ Sokoto } & \multicolumn{2}{|c|}{ Zamfara } \\
\hline & Freq & $\%$ & Freq & $\%$ & Freq & $\%$ & Freq & $\%$ & Freq & $\%$ & Freq & $\%$ & Freq & $\%$ \\
\hline Head & 81 & 7.53 & 14 & 2.35 & 53 & 4.12 & 25 & 15.15 & 11 & 1.15 & 9 & 0.95 & 15 & 1.59 \\
\hline Wife & 873 & 81.13 & 543 & 91.11 & 1129 & 87.72 & 100 & 60.61 & 903 & 94.75 & ltid96.9 & 96.93 & 915 & 96.83 \\
\hline $\begin{array}{l}\text { Daughter and others } \\
\text { Occupation type }\end{array}$ & 122 & 11.34 & 39 & 6.54 & 105 & 8.16 & 40 & 24.24 & 39 & 4.09 & 20 & 2.12 & 15 & 1.59 \\
\hline Unemployed & 474 & 44.05 & 234 & 39.26 & 387 & 30.07 & 308 & 30.92 & 361 & 37.88 & 504 & 53.33 & 323 & 34.18 \\
\hline Skilled and Unskilled & 182 & 16.91 & 31 & 5.20 & 337 & 26.18 & 102 & 10.24 & 80 & 8.39 & 135 & 14.29 & 93 & 9.84 \\
\hline Agric sector & 16 & 1.49 & 39 & 6.54 & 9 & 0.70 & 24 & 2.41 & 51 & 5.35 & 1 & 0.11 & 10 & 1.06 \\
\hline Service sector & 404 & 37.55 & 292 & 48.99 & 554 & 43.05 & 562 & 56.43 & 461 & 48.37 & 305 & 32.28 & 519 & 54.92 \\
\hline Total & 1076 & 100 & 596 & 100 & 1287 & 100 & 996 & 100 & 953 & 100 & 945 & 100 & 945 & 100 \\
\hline
\end{tabular}

Source: Authors' computations from the 2013 DHS data.

Table 4. Distribution of Rural Women by their Health and Nutrition status.

\begin{tabular}{lll}
\hline Category & Frequency & Per cent \\
\hline Ante natal care & & \\
Skilled Attendant & 956 & 14.06 \\
Non Skilled Attendant & 5842 & 85.94 \\
Assistance during delivery & & \\
Skilled Attendant & 273 & 4.02 \\
Non Skilled Attendant & 6525 & 95.98 \\
Place of delivery & & \\
Health facility & 340 & 5.00 \\
Non health facility & 6458 & 95.00 \\
Body Mass Index & & \\
Normal & 4975 & 73.18 \\
Abnormal (Thin and Obese) & 1823 & 26.82 \\
\hline
\end{tabular}

Source: Authors' computations from the 2013 DHS data.

Table 5. Distribution of Rural women by Autonomy.

\begin{tabular}{lll}
\hline Category & Frequency & Per cent \\
\hline $\begin{array}{l}\text { Decision maker on how to spend respondent's } \\
\text { earnings }\end{array}$ & & \\
Respondents alone & 3665 & 53.91 \\
Respondents and Partner & 190 & 2.79 \\
Husband/partner alone & 2932 & 43.18 \\
Someone else and others & 11 & 0.16 \\
Decision maker on own health & & \\
Respondents alone & 45 & 0.66 \\
Respondents and Partner & 762 & 11.21 \\
Husband/partner alone & 5978 & 87.94 \\
Someone else and others & 13 & 0.19 \\
Decision on large household purchases & & \\
Respondents alone & 38 & 0.56 \\
Respondents and Partner & 680 & 10.00 \\
Husband/partner alone & 6062 & 89.17 \\
Someone else and others & 18 & 0.27 \\
Decision on visit to family/relatives & & \\
Respondents alone & 99 & 1.46 \\
Respondents and Partner & 1226 & 18.03 \\
Husband/partner alone & 5454 & 80.23 \\
Someone else and others & 19 & 0.28 \\
\hline
\end{tabular}

Source: Author's computations from the 2013 DHS data.
Table 6. Distribution of Rural Women by their deprivation Index.

\begin{tabular}{lll}
\hline Deprivation Index & Frequency & Per cent \\
\hline $0.0000-0.1000$ & 148 & 2.18 \\
$0.1001-0.2000$ & 1167 & 17.17 \\
$0.2001-0.3000$ & 2371 & 34.88 \\
$0.3001-0.4000$ & 1896 & 27.89 \\
$0.4001-0.5000$ & 761 & 11.19 \\
$0.5001-0.6000$ & 324 & 4.77 \\
$0.6001-0.7000$ & 112 & 1.65 \\
$0.7001-0.8000$ & 18 & 0.26 \\
$0.8001-0.9000$ & 1 & 0.01 \\
$0.9001-1.000$ & 0 & 0.00 \\
Total & 6798 & 100 \\
\hline
\end{tabular}

Source: Authors' computations from the 2013 DHS data.

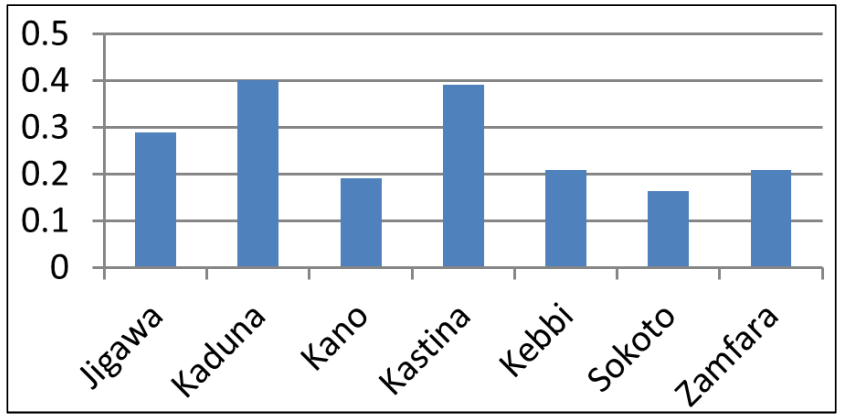

Source: Authors' computations from the 2013 DHS data.

Figure 1. Welfare deprivation Index across states.

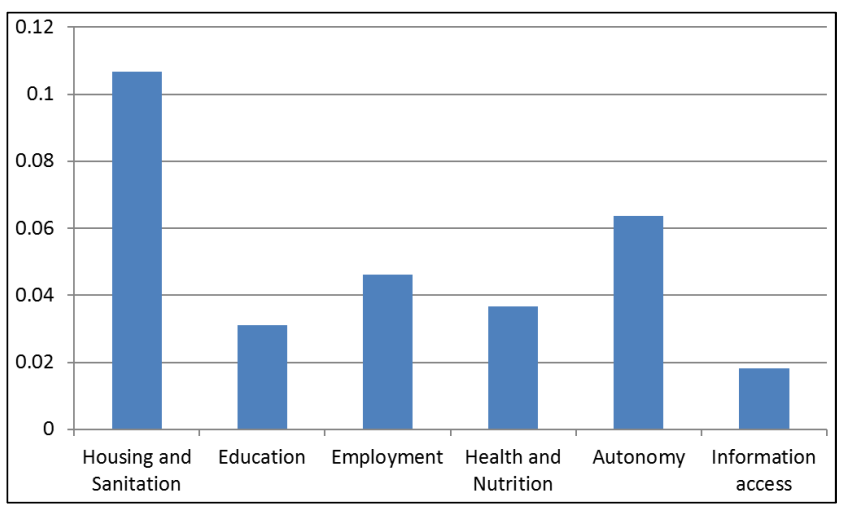

Source: Author's computations from the 2013 DHS data.

Figure 2. Multidimensional Deprivation Decomposition across Dimensions and Indicators. 


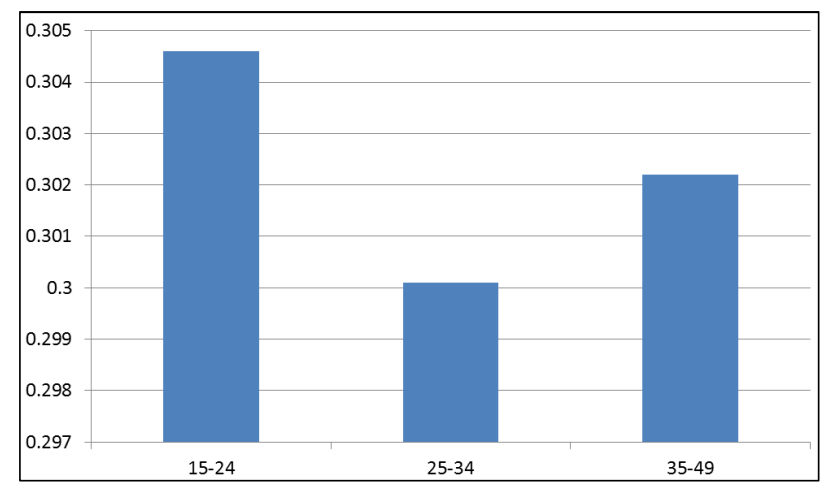

Source: Author's computations from the 2013 DHS data.

Figure 3. Multidimensional Deprivation Decomposition across Age.

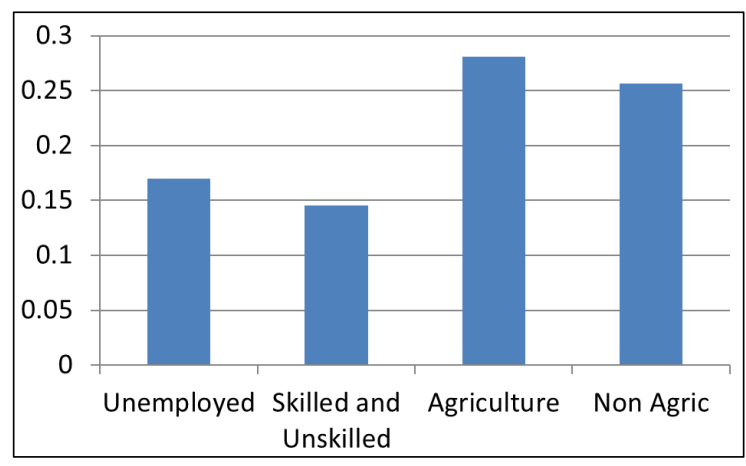

Source: Author's computations from the 2013 DHS data

Figure 4. Multidimensional Deprivation Decomposition across Employment type.

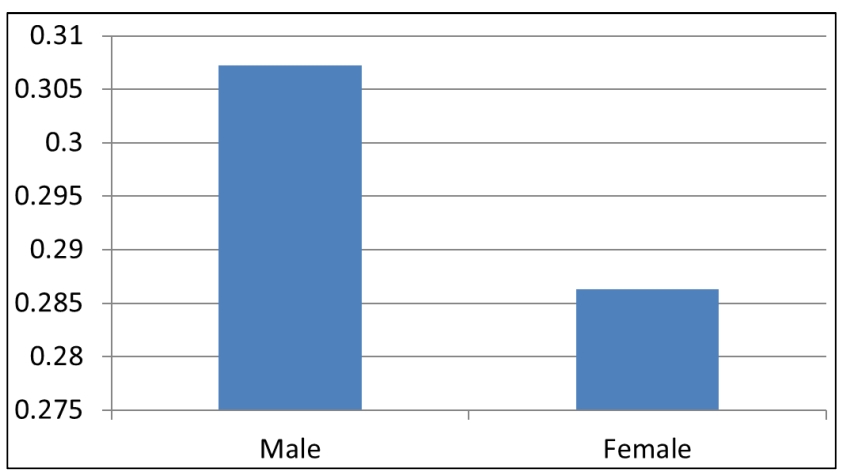

Source: Authors' computations from the 2013 DHS data.

Figure 5. Multidimensional Deprivation Decomposition across Gender of household head.

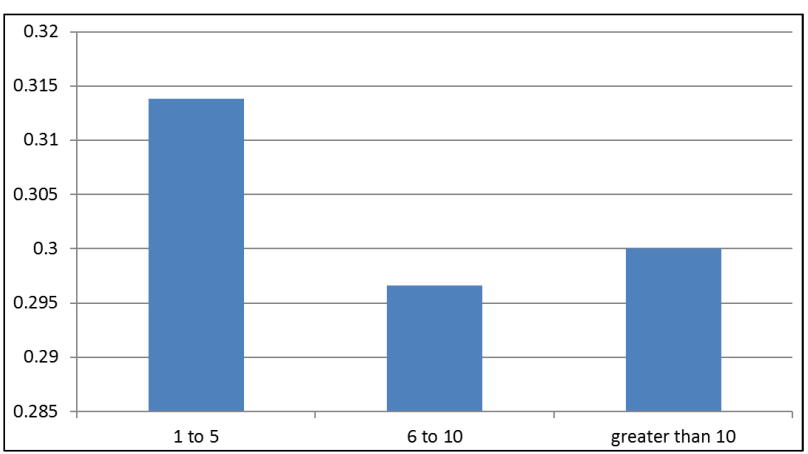

Source: Author's computations from the 2013 DHS data.

Figure 6. Multidimensional Deprivation Decomposition across Household size.

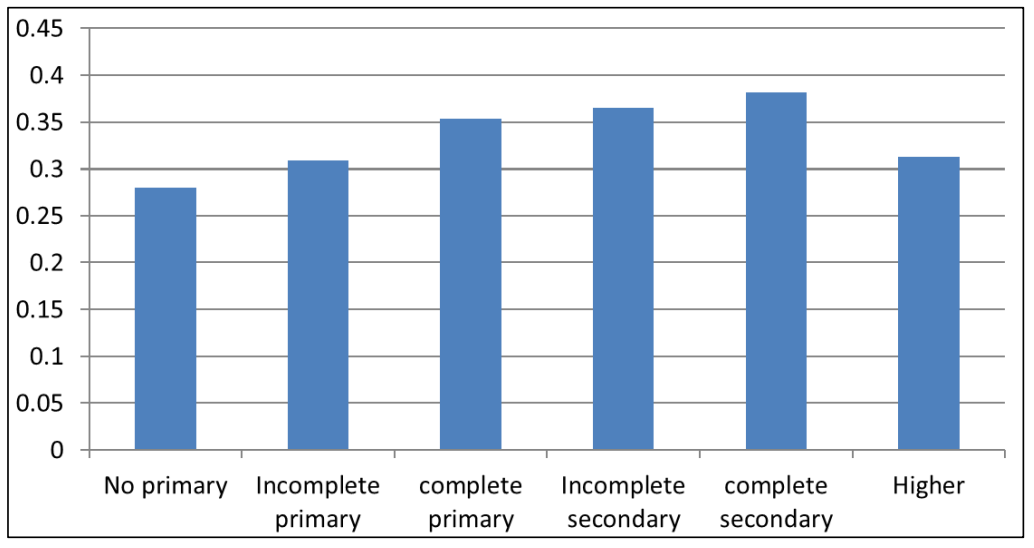

Source: Authors' computations from the 2013 DHS data.

Figure 7. Multidimensional Deprivation Decomposition across Educational group.

Table 7. Factors influencing asset ownership by women.

\begin{tabular}{|c|c|c|c|}
\hline Predictor variables & Coefficients & Standard error & Marginal effects \\
\hline \multicolumn{4}{|l|}{ Individual level factors } \\
\hline Age of women (b: $15-24$ years) $25-34$ years & 0.0305 & 0.0749 & 0.0055 \\
\hline $35-49$ years & $0.3096^{* * *}$ & 0.0784 & $0.0565 * * *$ \\
\hline $\begin{array}{l}\text { Employment (b: unemployed) } \\
\text { Skilled and Unskilled }\end{array}$ & $-1.5955 * * *$ & 0.0889 & $-0.3283 * * *$ \\
\hline Agric and allied & $-1.1332 * * *$ & 0.1842 & $-0.2315 * * *$ \\
\hline Services & $-2.1829 * * *$ & 0.0699 & $-0.4396 * * *$ \\
\hline $\begin{array}{l}\text { Educational attainment (b: no education) } \\
\text { Incomplete primary }\end{array}$ & $-0.5566 * * *$ & 0.1724 & $-0.1002 * * *$ \\
\hline Complete primary & $-0.2190 *$ & 0.1208 & $-0.0401 *$ \\
\hline
\end{tabular}




\begin{tabular}{|c|c|c|c|}
\hline Predictor variables & Coefficients & Standard error & Marginal effects \\
\hline Incomplete secondary & $-0.5685 * * *$ & 0.1997 & $-0.1022 * * *$ \\
\hline Complete secondary & -0.2617 & 0.2071 & -0.0479 \\
\hline Higher & -0.1609 & 0.4599 & -0.0295 \\
\hline \multicolumn{4}{|l|}{ Household level factors } \\
\hline $\begin{array}{l}\text { Gender of household head (b: Male) } \\
\text { Female }\end{array}$ & -0.3723 & 0.3156 & 0.0674 \\
\hline $\begin{array}{l}\text { Marital status (Single) } \\
\text { Married }\end{array}$ & $1.8186^{* * *}$ & 0.2349 & $0.2859 * * *$ \\
\hline $\begin{array}{l}\text { Household size (1-5) } \\
6-10\end{array}$ & $-0.2215 * * *$ & 0.0674 & $-0.0301 * * *$ \\
\hline$>10$ & $-0.5652 * * *$ & 0.0899 & $-0.0578 * * *$ \\
\hline \multicolumn{4}{|l|}{ Environmental factors (State: Sokoto) } \\
\hline Zamfara & $1.3994 * * *$ & 0.1089 & $0.2638 * * *$ \\
\hline Kastina & $1.9555^{* * *}$ & 0.1113 & $0.3684 * * *$ \\
\hline Jigawa & $0.8731^{* * *}$ & 0.1052 & $0.1612 * * *$ \\
\hline Kano & $-0.3119 * * *$ & 0.1059 & $-0.0514 * * *$ \\
\hline Kaduna & $1.3809 * * *$ & 0.1267 & $0.2602 * * *$ \\
\hline Kebbi & $0.2993 * * *$ & 0.1082 & $0.0529 * * *$ \\
\hline Constant & $-0.9430 * * *$ & 0.4027 & \\
\hline Log likelihood & -3715.989 & & \\
\hline Chi square & 1938.67 & & \\
\hline Probability & 0.0000 & & \\
\hline Pseudo R square & 0.2069 & & \\
\hline
\end{tabular}

Source: Author's computations from the 2013 DHS data.

*** $\mathrm{P}<0.01$ significant at $1 \%,{ }^{* *} \mathrm{P}<0.05$ significant at $5 \%,{ }^{*} \mathrm{P}<0.1$ significant at $10 \%$.

\section{Discussion}

\subsection{Socio Economic Characteristics}

In table $2,34.47 \%$ of women fall within the age range of 25-34 followed by those within the age range of 35-49 (33.63 per cent) in North West (NW) Geopolitical zone. The mean age of women is 30 with a standard deviation of 9. This implies that we have more middle aged women in rural NW and this may be because women in this zone marry early. Majority of the women in this zone no educational attainment $(85.80 \%)$. Women that had complete primary education were $6.38 \%$ while $2.06 \%$ had complete secondary education. According to the universal basic education that recommends that a girl child should have a minimum of nine years of education, based on this $11.21 \%$ of women in the southern region are educated. Women in this region have a mean household size of 7 . Most of the women are married $(97.63 \%)$. Women that are employed in the service sector are $45.56 \%$ followed by those that are unemployed (38.11\%) with $14.12 \%$ employed in the agriculture and allied sector. Most women in this region belong to a male headed households $(91.69 \%)$ while only 2.87 percent are heads of households.

All the states in NW had a mean age of between 30 years in table 3, except Jigawa, Kaduna and Zamfara. Also all the states has a mean household size of 7 members except Kano that has a mean of eight members. Sokoto state has the highest percentage of women with no education (94.50\%) while Kastina state has the highest percentage of women with complete primary education (11.55 percent). Based on the data used for this study Kano state has the highest number (1287) of women while Kaduna has the lowest number (596) in NW.
As shown in table 4, 85.94\% of rural women in NW receive antenatal care from non skilled attendant. This is common in rural setting where most rural areas do not have health centres or even where one exists, there are no health personnel to attend to patients or rural women may have to trek long distances in search of medical care. Also, only $5.00 \%$ of women deliver in health facilities. Only $4.02 \%$ of women were assisted by skilled health personnel during delivery. In spite of government efforts to achieve the SDGs, rural women largely patronise traditional birth attendants and some even prefer to deliver at home without assistance. Little wonder why maternal mortality is on the increase in the rural areas. Women in general are disadvantaged in accessing health services which have always resulted in high sickness rate and mortality. In Nigeria, there are over 7 million live births annually, Only $61 \%$ of pregnant Nigerian women attend the WHO-recommended for ante natal care visits, and less than $60 \%$ of births are attended by a skilled birth attendant [11].

Table 5 shows that highest percentage of women (53.91\%) take decisions alone in spending money while $43.18 \%$ of women's husband or partner decide on how they spend their money which is in line with the report given by $[1,2]$ that a larger percentage of women have autonomy on money spending. Observably, the distribution shows that making large household purchases, decision on own health and visits to friends and relatives representing $89.17 \%, 87.94 \%$, and $80.23 \%$, respectively are taken by their husbands or partners.

\subsection{Multidimensional Welfare Deprivation of Women}

Table 6 shows the distribution of rural women on their Deprivation status. The DI for rural women ranges from 0.01 to 0.82 with a mean value of 0.30 . On the average, women in 
rural NW Nigeria have DI between 0.2001-0.4000, thus are deprived this is in line with studies using unidimensional and multidimensional approach carried out in Nigeria [4] where women are believed to have low wellbeing. Using a multidimensional approach, the result is more pronounced with a larger number of women found to be worse off [2]. Figure 1 shows the deprivation index across states in rural North West.

The contribution of each welfare dimension to women's deprivation is presented in Figure 2. Among the six dimensions considered, housing and sanitation had the highest absolute and relative contributions of $0.11 \%$ and $35.26 \%$ and thus contributes the least to deprivation. This is followed by autonomy with $0.06 \%$ and $21.04 \%$. This means that rural women are better off in these dimensions than others. The high relative contribution of housing is expected since most of them live in the same house with their spouses. These houses are provided by the joint effort of the household. It is also worthy of note that autonomy has a high relative contribution. The high relative contribution of autonomy underscores the point that power relations within the household is crucial and ability to participate in decision making particularly with respect to self is important for women's well-being.

The lowest absolute and relative contributions of $0.02 \%$ and $6.05 \%$ respectively are recorded in information access and 0.03 and 10.32 in education these dimensions contributes more to deprivation. It implies that rural women's access to information and employment is poor presently and improving this dimension will improve their welfare. In ascending order of contribution, the six dimensions considered are arranged as follows: information access, education, health and nutrition, employment, autonomy, housing and sanitation. In view of the high deprivation index of women in general, these dimensions need to be improved on particularly information access, education, health and nutrition whose contributions to deprivation are high. The Levene's test shows that the variances of multidimensional well- being indices across dimensions are significantly different $(\rho=$ $0.0000)$.

\subsection{Decomposition Across Socio-Economic Groups}

In Figures 3-7, the decomposition of DI across socioeconomic characteristics of rural women is presented. These characteristics are age, household size, gender of household head, employment type, and educational attainment. The decomposition by age group presented in Figure 3 shows that middle aged women within the age group of 25 to 34 years have lower DI compared to other age groups.

The absolute and relative contributions obtained for employment type is presented in Figure 4. It reveals that women that are skilled and unskilled worker have lower DI (0.1451) compared to unemployed (0.1701), those employed in the non-agricultural sector $(0.2565)$ and those in the agricultural sector (0.2809). This might be because skilled and unskilled worker are mostly paid daily, and thus have money to spend per day unlike their counterparts employed in other sectors.

In Figure 5, the deprivation indices of women in male and female headed households are 0.3072 and 0.2863 respectively. Women in male headed household have a lower DI than those in female headed households.

With respect to household size (Figure 6), the subgroup belonging to small household size (6 to 10) has lower DI than other groups. This subgroup has a 0.2966 DI, followed by those with greater than 10 household size with 0.3000 DI.

The deprivation indices across educational groups increase from no education to complete secondary and decreases at higher education level. (Figure 7). The peak is attained by the group with complete secondary while the least is recorded in the group with no education.

In summary, middle aged women with higher education from a medium sized female headed household, skilled and unskilled worker have lower deprivation indices than other groups. The Levene's test show that the variances of multidimensional wellbeing indices across socio-economic characteristics of rural women are significantly different (= 0.0000).

\subsection{Determinants of Asset Ownership by Women}

The result of the regression analysis is presented in Table 7. The columns present the coefficients and their marginal effects. The diagnostic statistics reveal that the model has a log likelihood ratio of -3715.989 and a chi-square statistic of 1938.67; significant at one per cent. This shows that the model is a good fit for the data. Sixteen variables were found to significantly influence welfare deprivation.

Individual level factors

The coefficient of the age group 35 to 49 years is 0.3096 , indicating that a change in the age category from the base category (15 to 24 years) to 35 to 49 years category significantly increase the deprivation status of the latter. The $\log$ odds of the latter being 0.31 times that of the former, older women (35 to 49 years) are more likely to have high deprivation status compared to younger ones (15 to 24 years). The marginal effect estimates reveal that being in the age group 35 to 49 years increase the likelihood of being deprived by $5.7 \%$.

With regards to employment type, all the employment types significantly influence deprivation status at one per cent. The results reveal that being employed in any of these sectors is likely to reduce the probability of deprivation. Although the non- agricultural sector has a higher coefficient than others. Women in all the employment types are likely to experience lower deprivation.

Also, with regards to the educational attainment of women, there is a negative relationship with deprivation. Levels of educational attainment from incomplete primary education to incomplete secondary education are significant at one to ten per cent in reducing deprivation of women. Also, from the estimated coefficients, incomplete secondary education has the highest estimate (0.57) which shows that if a women is educated to incomplete secondary education level, the lower the probability of deprivation. 
The results show that educational attainment exerts high marginal impacts on deprivation. There is a gap between having a complete primary and secondary school education as shown by a difference of 0.01 in the marginal effects. The implication is that, for a meaningful improvement in the welfare of women in rural NW, the completion of a primary school education is the required minimum.

Household level factors

Being a married woman will increase the probability of being deprived compared to being single. The results show a positive relationship between deprivation status of women and being married. Being a married woman in rural NW increase the probability of deprivation by 0.29 . This might be because of low autonomy they have on final say on their own health and final say on making large household purchases.

Women in medium to large households are more likely to have low deprivation status compared to women in small households. In other words, family size is inversely related to women's deprivation status. The marginal estimates reveal a 0.0578 increase in the probability of being better off in households with greater than ten people.

Regional factor

When compared with the Sokoto, rural women in Kano are better off which reveals a negative relationship with higher levels of deprivation and is significant at one percent. Meanwhile the six remaining states have a positive relationship with deprivation thus they are worse off in deprivation. In summary, educational attainment, employment type, household size, marital status, age of the woman, state of residence determines a woman's deprivation status in rural NW.

\section{Conclusion}

This study provides empirical evidence of the welfare deprivation of women in the North-West. Interventions in the area of information access, education, health and nutrition, is needed for women in North-West. More efforts by agencies working with women are needed for women in the NW through empowerment programs that take into cognizance their peculiarities such as soap and cream making, knitting of mat and bags to improve their welfare status. Government and Non-Government organisations should put in place policies to improve education at the grassroots for women.

\section{References}

[1] Adeoti, A. and A. O. Oni. (2009). Demand for reproductive health and child mortality, in Nigeria. www.poppov.org/Portals/1/documents/papers/26.Adeoti.pdf.

[2] Adeoti A. O and Akinwande B (2013): Poverty and Well being of Women in rural Nigeria. LAP LAMBERT Academic Publishing. VDM Verlagsservicegesellschaf.

[3] Agbodji A. E., Maweki Y., Ouedraogo B. D, (2015). "Gender inequality in multidimensional welfare deprivation in West Africa", International Journal of Social Economics, 42 (11),
$980-1004$.

[4] Alaye-Ogan, E. O. (2008). Rural poverty among women in Nigeria: A case study of Abuja satellite communities of Nigeria. A Dissertation submitted to the School of Post Graduate Studies, t Clements University, Turks \& Caicos Islands, British West Indies in partial fulfillment of the requirements for the award of a degree of Doctor of Philosophy in Development Economics.

[5] Cook, S. (2013). Combating poverty and inequality: What role for social protection? Paper presented at the Asia Public Policy Forum, Jakarta, Indonesia May 28-30.

[6] Day-Hookoomsing, P. (2002). Women and the New World economy: an island's experience. Women in Management Review, 17 (7), 308-317.

[7] Gyimah-Brempong, K. (2002). Corruption, economic growth, and income inequality in Africa. Economics of Governance, 3 (3), 183-209.

[8] Majumder, A. (2006). The state and plight of Indian women: a multidimensional assessment of well-being based on Sen's functioning approach. In International Conference of the uman Development and Capability Association: Freedom and Justice, September, Groningen, the Netherlands.

[9] Martinetti E. C (2000). A Multidimensional Assessment Of Wellbeing Based On Sen's Functioning Approach. Rivista Internazionale di Scienze Sociali, 108, 207-239.

[10] Morrison, D. (1971). Some Notes Toward Theory on Relative Deprivation, Social Movements, And Social Change. The American Behavioral Scientist, 14 (5), 675.

[11] National Bureau of Statistics. (2012). The Nigeria poverty profile 2010 report.

[12] NDHS (2013). Nigeria Demographic and Health Survey.

[13] Ologbon O. A. C., Adeoti A. I., Omonona B. T. and Falusi A. O (2012). Welfare Deprivation Among Riverine Households In Southwestern Nigeria. ARPN Journal of Agricultural and Biological Science, 7 (5), 330-341.

[14] Oni, O. A. and Adepoju, T. A. (2011). A Capability Approach to the Analysis of Rural Households' Wellbeing In Nigeria. MPRA Paper No. 34508.

[15] Oyekale, A. S and Okunmadewa, F. Y. (2008). Fuzzy Set Approach to Multidimensional Poverty Analysis in Abia State, Nigeria. Journal of Applied Sciences, 3 (7), 490-495.

[16] Pampalon, R., and Raymond, G. (2000). A deprivation index for health and welfare planning in Quebec. Chronic Diseases and Injuries in Canada, 21 (3), 104.

[17] Rasaki S. D (2016): Poverty and Economic Growth in Nigeria: Issues and Policies, Journal of Poverty, DOI: 10.1080/10875549.2016.1141383.

[18] Salmond, C. E., Crampton, P., and Atkinson, J. (2007). NZ Dep 2006 index of deprivation.

[19] Townsend, P. (1979): Poverty in the United Kingdom. A Survey of Household Resources and Standards of Living. Harmondsworth, Penguin Books.

[20] Townsend P. (1987). Deprivation, Journal of Social Policy, 16 (2), 125-146. 